\title{
Topology is destiny: Reconnection energetics in the corona
}

\author{
D. W. Longcope and C. C. Kankelborg \\ Department of Physics, Montana State University, Bozeman, MT 59717, U.S.A. \\ (Received June 15, 2000; Revised August 21, 2000; Accepted October 2, 2000)
}

\begin{abstract}
Magnetic reconnection is clearly at work in the solar corona reorganizing and simplifying the magnetic field. It has also been hypothesized that this reorganization process somehow supplies the energy heating the corona. We propose a quantitative model relating the topological role (simplification) and the energetic role (heating) of magnetic reconnection. This model is used to analyze multi-wavelength observations of an X-ray bright point. In the model, motion of photospheric sources drives reconnection of coronal flux. If reconnection occurs only sporadically then energy is stored in the coronal field, and released by topological reconnection. We simulate the dynamical response of the plasma to such an energy release, and translate this into predicted observational signatures. The resulting predictions are difficult to reconcile with the observations. This suggests that while reconnection is important in the corona, energy dissipation is governed by other factors, not all of which relate to the topology of the field.
\end{abstract}

\section{Reconnection in Two Dimensions-a Concep- tual Framework}

Until recently, the majority of theoretical work on magnetic reconnection has been based on two-dimensional models. Some two-dimensional models have shown slow reconnection rates, most notably the scaling of Sweet and Parker (Sweet, 1958; Parker, 1957); while other models have demonstrated reconnection at the maximum rate allowable by the magnetic field (Petschek, 1964; Sonnerup, 1970; Hassam, 1992; Craig and McClymont, 1993), a situation known as fast reconnection. These conflicting results can largely be attributed to different versions of Ohm's law in the models. Fully non-linear solutions using a uniform resistivity typically exhibit slow reconnection occurring at an elongated current layer (Biskamp, 1986). Non-uniform (anomalous) resistivity (Ugai and Tsuda, 1977; Scholer and Roth, 1987) or non-resistive terms in Ohm's law (Biskamp et al., 1997; Shay et al., 1998), can lead to fast reconnection occurring at a compact X-point.

In spite of their differences, most two-dimensional reconnection models can be understood in terms of a common global picture whose key landmarks are indicated in Fig. 1 (Craig, 1994). The common starting point is an equilibrium magnetic field containing an X-point, where both planar components of the field vanish (Dungey, 1958; Chapman and Kendall, 1963). Among its many defining characteristics the X-point lies at the interface of four topologically distinct domains of flux. The domains are identified by the positive and negative boundary regions they connect: $P 1-N 1$ etc. In the sense that reconnection changes the topology of field lines it must occur at the $\mathrm{X}$-point, where all four topologies meet.

Reconnection will result when this equilibrium is per-

Copy right $(\mathrm{C}$ The Society of Geomagnetism and Earth, Planetary and Space Sciences (SGEPSS); The Seismological Society of Japan; The Volcanological Society of Japan; The Geodetic Society of Japan; The Japanese Society for Planetary Sciences. turbed in some way. Here we consider a perturbation driven from a distant boundary, away from the X-point itself. In cases of slow reconnection, or no reconnection at all, the flux in each of the four topological domains must remain fixed. The new magnetic equilibrium accessible under this constraint contains a current sheet at the location of the $\mathrm{X}$ point (Green, 1965; Syrovatskii, 1971); this is equilibrium $Y$.

Changing the flux in any domain requires an electric field in the ignorable direction. The common feature of all reconnection models is that such an electric field occurs even at the X-point where the ignorable component of $\mathbf{v} \times \mathbf{B}$ vanishes. With the possibility of topological change, the field can assume its state of absolute minimum energy (subject to boundary conditions): state $X^{\prime}$. In certain models of purely fast reconnection, known as X-point collapse models, the field proceeds directly from the perturbed state $X^{*} \rightarrow X^{\prime}$, bypassing $Y$ (Craig, 1994). Fast reconnection of the type we will consider, however, transfers flux through an existing current sheet (Ugai and Tsuda, 1977; Scholer and Roth, 1987; Heyn and Semenov, 1996), making possible the rapid transition $Y \rightarrow X^{\prime}$.

At the most fundamental level, the perturbation changes the external boundary conditions to make $X^{\prime}$ the minimum energy state. Since it does this without re-allocating domain fluxes, state $X^{\prime}$ is topologically inaccessible, and equilibrium $Y$ is the lowest accessible state. The energy difference

$$
\Delta E_{Y}=E_{Y}-E_{X^{\prime}},
$$

can be attributed to field topology alone (Craig, 1994).

The difference in domain fluxes, $\Delta \psi$, is the single relevant characteristic of the perturbation. The length $\Delta$ of the current sheet and the energy different $\Delta E_{Y}$ are functions of $\Delta \psi$ alone (Craig, 1994). To accomplish the $Y \rightarrow X^{\prime}$ transition magnetic reconnection must transfer a total flux $\Delta \psi$ from domains $P 1-N 2$ and $P 2-N 1$ into domains $P 1-N 1$ and $P 2-$ 

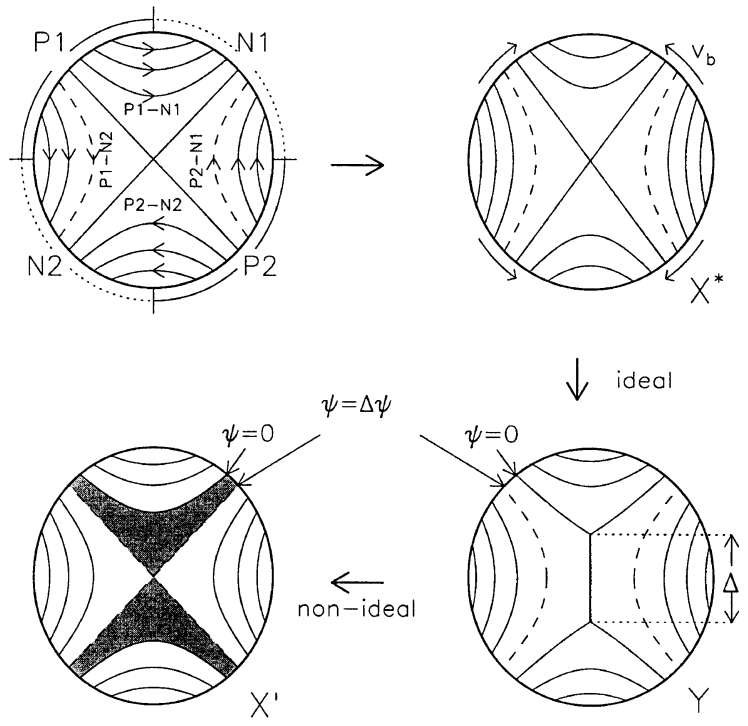

Fig. 1. The unifying framework for two-dimensional models of magnetic reconnection. From an initial equilibrium the field is perturbed into state $X^{*}$. The lowest-energy (ideally) accessible state from $X^{*}$ is state $Y$. The lowest-energy state, ignoring topological constraints, is $X^{\prime}$.

N2. The post-reconnection regions, shown shaded in Fig. 1, are the natural sites for energy $\Delta E_{Y}$ to be deposited.

\section{Extending to Three Dimensions}

The global scenario above has a direct three-dimensional analog (Sweet, 1958; Baum and Bratenahl, 1980; Greene, 1988; Lau and Finn, 1990; Longcope and Cowley, 1996). Consider a conducting boundary (the photosphere) at $z=0$, with four sources of flux, labeled $P 1, N 1, P 2$ and $N 2$ as shown in Fig. 2. The coronal field consists of four topologically distinct domains $P 1-N 1$ etc., exactly as in the twodimensional example. A vertical plane $M$ dividing positive from negative sources intersects the four domains in a pattern similar to that in two-dimensions. At the center of this pattern the plane intersects a field line called the separator. This single field line is the topological analog of the X-point of two-dimensions. Equilibrium $Y$, which minimizes magnetic energy subject to a constraint on all domain fluxes, will contain a singular current at the separator (Longcope, 1996; Longcope and Cowley, 1996). The most significant characteristic of equilibrium $Y$ is that domain $P 1-N 1$ contains magnetic flux different from a potential field by $\Delta \Phi$.

\section{An Example: Two Interacting Poles}

X-ray bright points (XBPs) provide one of the simplest observational signatures of magnetic reconnection in the solar corona. An XBP is formed when two photospheric sources of opposite polarity approach closely enough to undergo reconnection (Golub et al., 1977; Webb et al., 1993; Harvey et al., 1994; Kankelborg et al., 1996). Prior to their interaction, flux from each pole, $P 1$ and $N 1$, is connected to other sources. Modeling the other sources, e.g. $P 2$ and N2 in Fig. 2, as a uniform horizontal background magnetic field $B_{0}$, the flux from each pole is encompassed by a separatrix surface (see Fig. 3). When the separatrix surfaces do not intersect (Fig. 3(a)) there are three flux domains: one in-

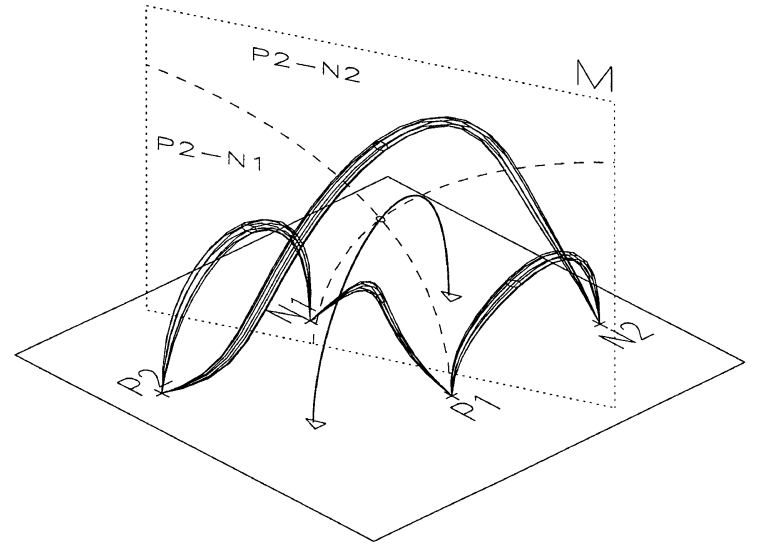

Fig. 2. Three-dimensional analog of Fig. 1. A few field lines from each domain are shown. The domains intersect plane $M$ in an X-point pattern.
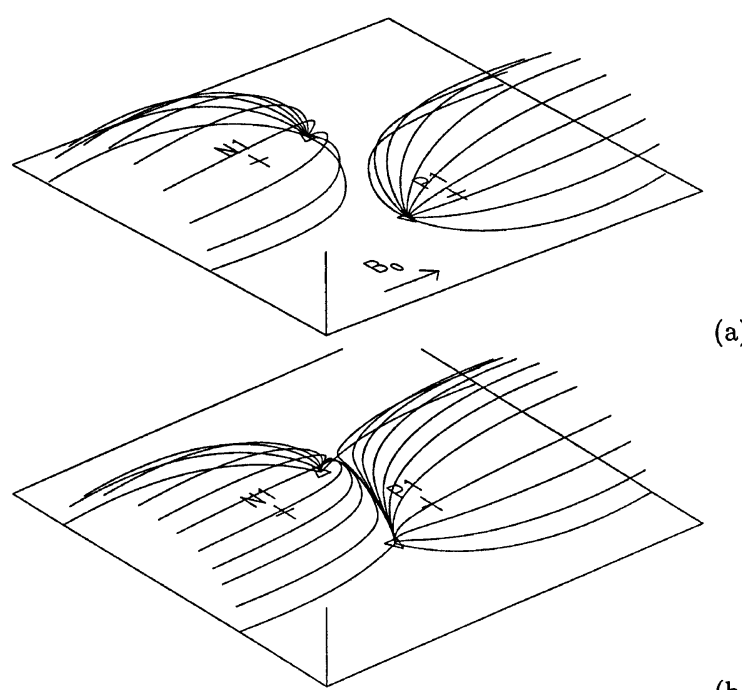

(a)

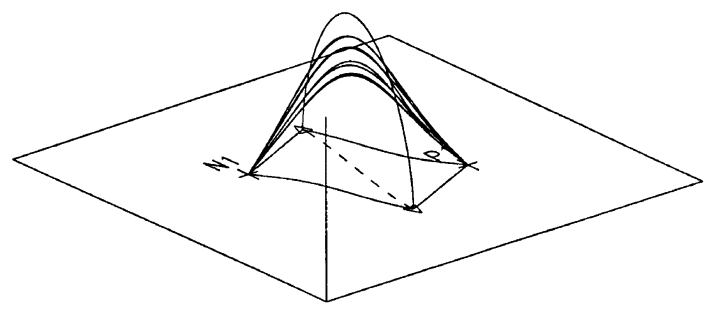

(b)

(c)

Fig. 3. Reconnection between a pair of opposing sources. (a) Separatrices enclosing the two flux domains prior to interaction. (b) Interaction between the two sources, indicated by an intersection of the separatrices. (c) The separator and a few of the post-reconnection field lines in domain $P 1-N 1$. External sources, e.g. $P 2$ and $N 2$ lie outside the figure.

side each separatrix and a single background domain outside. Once the sources approach to within the interaction distance $\sim D=\sqrt{\Phi / B_{0}}$ (Longcope, 1998) the two separatrix surfaces intersect along the separator. In the interacting case there is one new domain, $P 1-N 1$, consisting of field lines which connect the two sources. This new domain is properly termed the bipole.

Progressing from Fig. 3(a) with three flux domains to 
Fig. 3(b) with four domains requires topological reconnection. Flux from domains $P 1-N 2$ and $P 2-N 1$, must be topologically changed to form additional background flux $(P 2-$ $N 2)$ and new bipole flux $(P 1-N 1)$. The reconnection must occur at the separator, where all four domains meet (Lau and Finn, 1990; Longcope, 1996). The newly forged (postreconnection) field lines will lie inside domain $P 1-N 1$, very near the separator.

In quiet Sun regions, XBPs are visible in soft X-rays or EUV emission as small ( $\lesssim 60 \mathrm{Mm})$ isolated features, often single loops (Golub et al., 1974). According to some theories, energy radiated is supplied to the XBP by magnetic reconnection (Priest et al., 1994; Longcope, 1998). In terms of the framework described above, this means that the process of flux transfer into domain $P 1-N 1$ must be supplying energy to the coronal plasma. We adopt here the model that the energy released by reconnection is the topological energy $\Delta E_{Y}$. We also assume that the energy is dissipated in the post-reconnection flux at a rate comparable to the rate of flux transfer (i.e. on times comparable to the Alfvén transit time).

\section{Modeling an Observation}

The TRACE spacecraft made multi-wavelength observations of a quiet Sun region on June 17, 1998, in which the evolution of a single XBP, located very near disk center, is particularly well documented. Synoptic SOI/MDI full-disk magnetograms, made every $96 \mathrm{~min}$, show the XBP to be associated with magnetic poles called $P 01$ and N01. These observations and related analysis are described by Kankelborg and Longcope (1999) in extensive detail; we present here a brief summary of that work, focusing on reconnection energetics.

According to the 9:36 SOI/MDI observation (see Fig. 4), the poles are separated by $d=11.3 \mathrm{Mm}$ (in the tangent plane) and are converging steadily at $\dot{d}=218 \mathrm{~m} / \mathrm{sec}$. At the same time each pole has a magnetic flux of approximately $1.1 \times 10^{19} \mathrm{Mx}$, which is decreasing steadily at a rate of approximately $\dot{\Phi}_{1}=3.5 \times 10^{14} \mathrm{Mx} / \mathrm{sec}$, presumably by subduction.

To investigate the effects of this convergence and cancellation upon the corona, we use a simplified model of the magnetic field. In the photospheric field model, point sources representing poles $P 01$ and $N 01$ steadily approach each other and their fluxes steadily decrease, while the "spectator poles" (all poles other than $P 01$ and $N 01$ ) remain at positions and fluxes from the 9:36 observation. A uniform horizontal background field, $B_{0}=10 \mathrm{G}$ directed $65^{\circ}$ North of West, is added to reproduce the general context shown in the TRACE image (Kankelborg and Longcope, 1999).

In a potential field based on the above photospheric model, flux will be reconnected into the $P 01-N 01$ domain at a rate of $\dot{\Phi}=1.6 \times 10^{14} \mathrm{Mx} / \mathrm{sec}$. This is equivalent to a 1.6 Megavolt loop-voltage along the separator field line. A conducting corona will not permit such an electric field, so the flux discrepancy $\Delta \Phi$ increases with time.

Unreconnected flux $\Delta \Phi=\dot{\Phi} \Delta t$ will accumulate during a delay $\Delta t$ in magnetic reconnection. The minimum energy state for the unreconnected field, state $Y$, will contain a current $I=\Delta \Phi / \mathcal{L}$ along the separator, where $\mathcal{L} \simeq 10^{8}$ emu is

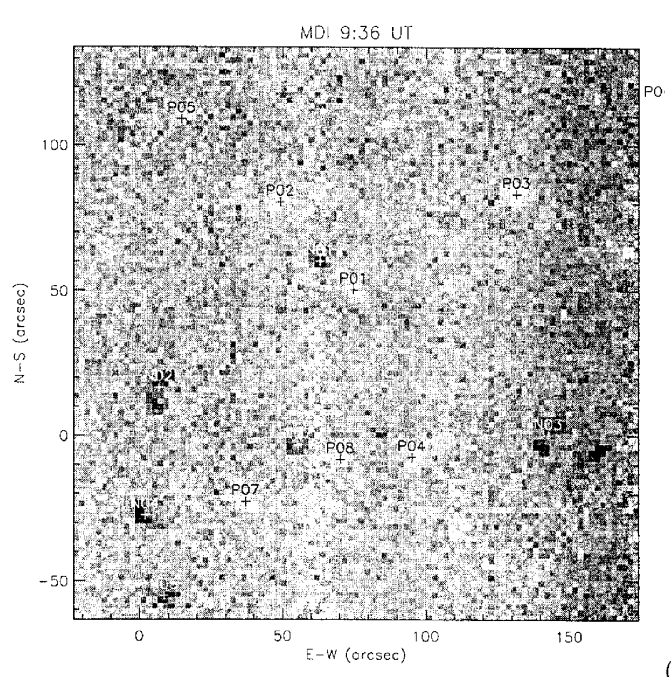

(a)

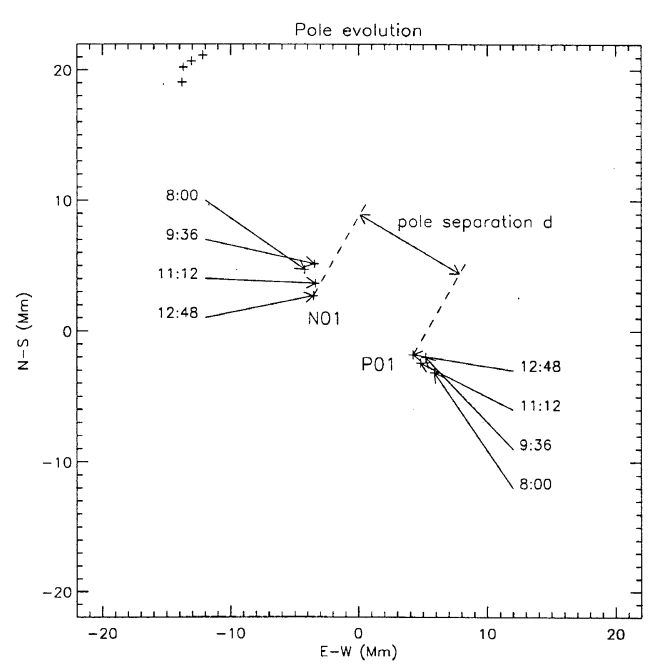

(b)

Fig. 4. Evolution of the magnetic sources observed by SOI/MDI. (a) A portion of the full-disk magnetogram showing flux sources including the bipole $P 01$ and N01. The motion of $P 01$ and $N 01$ during the time of the TRACE observation.

the self-inductance of the $25 \mathrm{Mm}$ long separator (Longcope, 1996)

From a strictly topological standpoint, any process which transfers flux $\Delta \Phi$ into domain $P 01-N 01$ will permit access to potential state, equilibrium $X^{\prime}$. The flux transfer thereby drops the configuration's minimum energy by

$$
\Delta E_{Y}=\frac{1}{2}(\Delta \Phi)^{2} / \mathcal{L}=10^{20}(\Delta t)^{2} \text { ergs }
$$

where $\Delta t$ is the time over which reconnection is inactive. Delaying reconnection for $\Delta t=20$ minutes will produce a flux-deficit $\Delta \Phi=1.8 \times 10^{17} \mathrm{Mx}$, and store $\Delta E_{Y}=1.6 \times$ $10^{26} \mathrm{ergs}$ in the equilibrium magnetic field.

We assume that energy $\Delta E_{Y}$ is released by a fast reconnection process, into the volume of plasma occupying the same $1.8 \times 10^{17}$ which is transferred across the separator. This flux is a tube located in domain $P 01-N 01$ just below the separator. Viewed from the same angle, the flux tube (Fig. 5(b)) is geometrically similar to the XBP observed by TRACE (Fig. 5(a)). We model the energy release as a steady plasma 


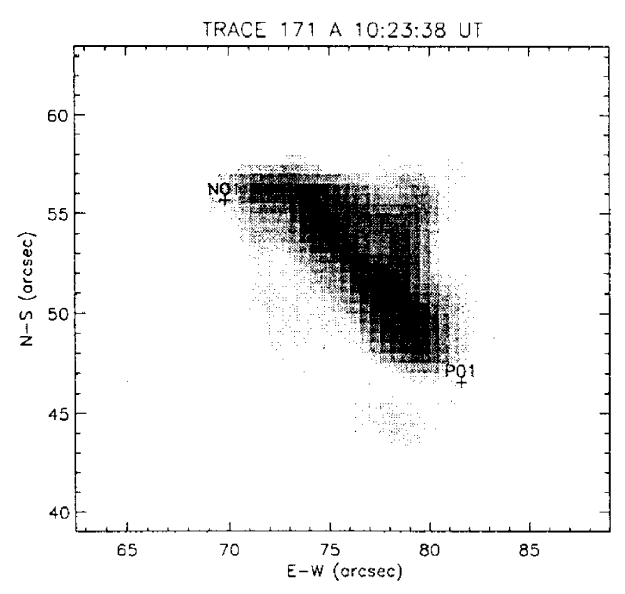

(a)

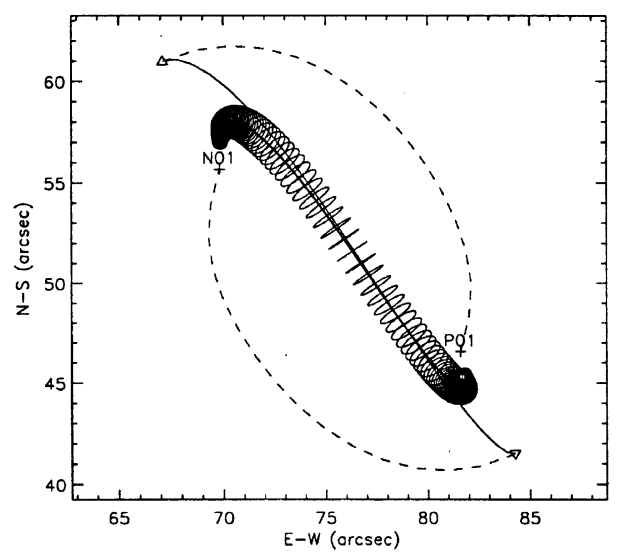

(b)

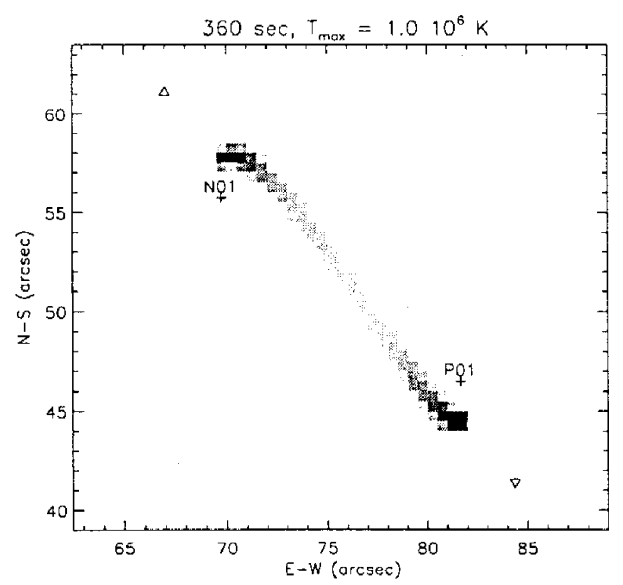

(c)

Fig. 5. Observations and model of an XBP. (a) The background-subtracted emission from TRACE $171 \AA$ channel during a period of intense XBP activity. (b) The outline of a post-reconnection flux tube of $\triangle \Phi=1.8 \times 10^{17}$ Mx immediately below the separator. Dashed lines show the base of the separatrices enclosing the entirety of domain P01-N01. (c) Plasma emission from a gas dynamics simulation passed through TRACE $171 \AA$ response.

heating with duration equal to ten Alfvén transit times. We calculate the dynamical response of the coronal plasma to this heating, and from that find the image which would be formed by each of the TRACE EUV channels (Fig. 5(c)).

A one-dimensional gas dynamic simulation (Kankelborg and Longcope, 1999) is run using the geometry defined by the

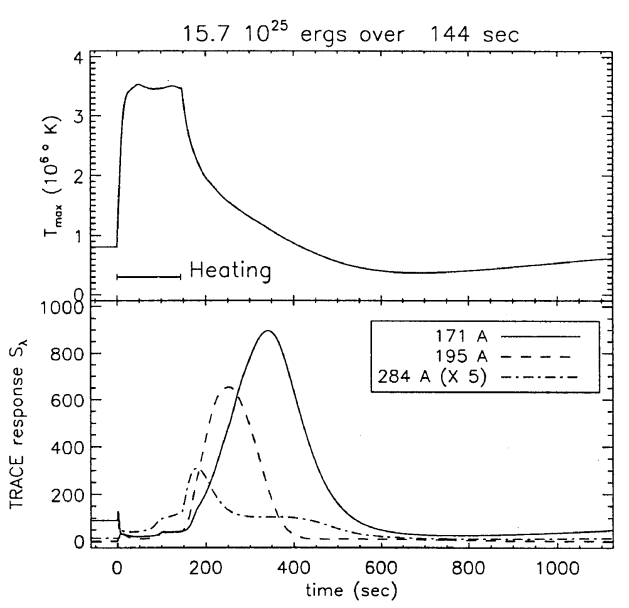

Fig. 6. Global characteristics of the gas dynamic simulation of the post-reconnection loop. (a) $T_{\max }$ over the simulation. (b) The integrated intensity observable in each of 3 TRACE pass-bands.

post-reconnection loop (namely the cross-sectional area $A(\ell)$ and the component of gravity parallel to the loop's axis, $\hat{\mathbf{l}} \cdot \mathbf{g})$. Time evolution of density, pressure and velocity are found from the continuity equation, parallel momentum equation, and energy equation including classical thermal conductivity and radiative losses. At each footpoint is a dense, cool chromosphere from which material naturally evaporates during the heating phase.

The simulation is first run to establish an initial equilibrium in the loop. A uniform "background" heating $q_{0}=7 \times 10^{-4}$ erg $\mathrm{cm}^{-3} \mathrm{sec}^{-1}$ (totaling $1.5 \times 10^{22} \mathrm{ergs} / \mathrm{sec}$ ) is applied and the equations are solved until they achieve a steady state. The steady state loop has $T_{\max }=800,000 \mathrm{~K}$, a total thermal energy of $E_{\mathrm{th}}=4 \times 10^{24} \mathrm{ergs}$, and an Alfvén transit time of $\tau_{\mathrm{A}}=14.4$ seconds.

At $t=0$ the heating is increased to $q=5 \times 10^{-2}$ erg $\mathrm{cm}^{-3} \mathrm{sec}^{-1}$ for 144 seconds $\left(10 \tau_{\mathrm{A}}\right)$ to simulate reconnection, and then returned to $q_{0}$. This deposits all $\Delta E_{Y}=$ $1.6 \times 10^{26}$ ergs of stored topological magnetic energy in a period intended to represent fast magnetic reconnection. The resulting temperatures and densities are used in conjunction with plasma emission models and the TRACE filter responses to produce the synthetic light curves shown in Fig. 6. Finally, lines of sight through the loop are used to synthesize an image as shown in Fig. 5(c)). A circular cross-section has diminishing line-of sight at its edge so the synthesized loop (Fig. 5(c)) appears narrower than the flux tube itself (Fig. 5(b)).

\section{Comparison with Observation}

The exercise above suggests the power heating an XBP does not come solely from magnetic reconnection narrowly defined. The most glaring inadequacy of the reconnection hypothesis is that it cannot produce an extended region of EUV emission similar to that observed by TRACE. Figure 5(a) shows the background-subtracted $171 \AA$ emission from a particularly active phase in the life of the XBP. This active phase lasted from 9:45 to 10:30 (Kankelborg and Longcope, 1999), and appears to cover a larger area than the model flux tube shown in Fig. 5(c).

The size discrepancy between observation and model 


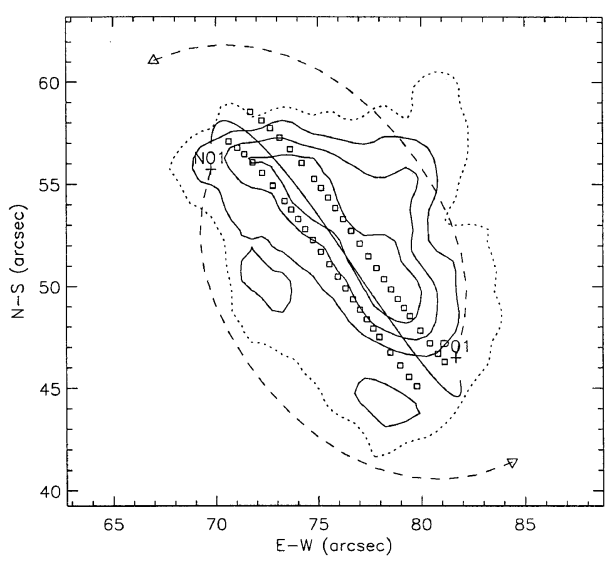

Fig. 7. Contours of the background-subtracted image, Fig. 5(a). Solid contours are $75 \%, 50 \%$ and $25 \%$ of maximum, dotted contour is $10 \%$. The axis of the model loop is shown at the center of a band 1".75 wide, marked by boxes. The base of the separatrix is denoted by dotted curves.

arises from our assuming fast reconnection and rapid (i.e. impulsive) energy release. The diameter of the model flux tube, $1.3 \mathrm{Mm}\left(1^{\prime \prime} .75\right)$ is set by the flux $\Delta \Phi=1.8 \times 10^{17}$ $\mathrm{Mx}$ and the field strength $B \simeq 14 \mathrm{G}$ along most of the separator. The flux is set, in turn, by the assumed delay preceding reconnection-for this we took the reasonable value $\Delta t=20 \mathrm{~min}$. Contours of the background-subtracted image Fig. 7, show that the brightest emission ( $\geq 75 \%$ of maximum) is indeed confined to a band of width comparable to the model. This lends support to our choice of $\Delta t$.

The $50 \%$ contours, on the other hand, trace a loop whose diameter, $\sim 4^{\prime \prime}$, corresponds to $10^{18} \mathrm{Mx}$ of post-reconnection flux. This represents 1.75 hours of flux at the inferred supply rate of $\dot{\Phi}=1.6 \times 10^{14} \mathrm{Mx} / \mathrm{sec}$. Choosing $\Delta t=1.75$ hours would produce a broader loop, however, with a substantial dead-time ( $\sim 1.5$ hours) between loop brightenings. The 8 hour observation showed little evidence for such dead-time (Kankelborg and Longcope, 1999). In short, our synthetic image Fig. 5(c) appears anemic simply because we assumed that reconnection would dissipate its energy in times much shorter than 1.75 hours $\left(\sim 400 \tau_{\mathrm{A}}\right)$.

It is possible that impulsively heated plasma would cover a larger apparent area if it were mixed with cooler, nonemitting plasma. There is observational evidence for this kind of coronal filling factor in coronal loops (Porter and Klimchuk, 1995; Cargill and Klimchuk, 1997). In a variant on the nanoflare model (Parker, 1988; Kopp and Poletto, 1993; Cargill, 1994) we perform the full flux transfer in $n$ smaller sub-transfers of $\Delta \Phi / n$ each. Each sub-loop is impulsively heated at a slightly different time, after which it undergoes evaporation, brightening and cooling according the gas-dynamic simulation. The resulting light-curve is a super-position of individual pulses like that in Fig. 6. Depending on resolution and the detailed timing of energy releases, the synthetic image shows multiple distinct loops, or one larger "compound loop" (see Fig. 8).

While the nanoflare model better resembles any given TRACE image, it continues to suffer from the drawbacks of the basic reconnection hypothesis. There are other points for comparison which cannot be discussed in this brief re-

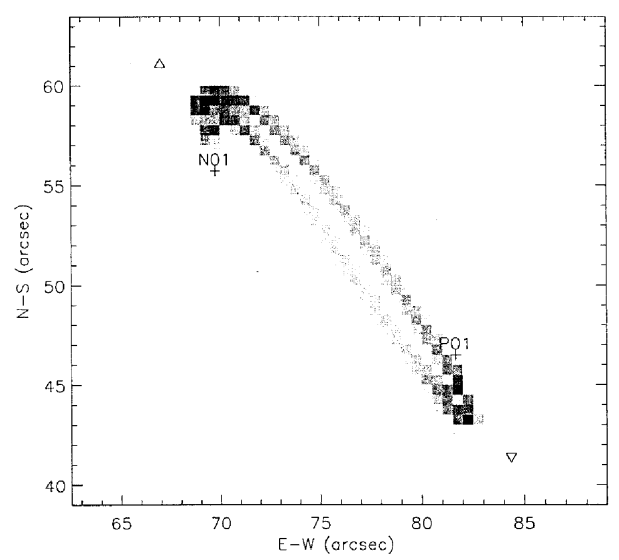

Fig. 8. A synthetic image from a nanoflare reconnection model (to enhance details, the gray scale shows square-root of intensity). The same flux from Fig. 5(c) is transferred in $n=10$ pieces, at random intervals over the course of $1200 \mathrm{sec}$.

view. For example, it is evident from the time history, Fig. 6, that the impulsive heating drives the plasma temperature to $>3 \mathrm{MK}$. All of TRACE's filters are sensitive to temperatures lower than this, and thus see the bulk of the loop only as it cools. This predicts that fluctuations in $171 \AA$ should lag those in $195 \AA$ by $\sim 100 \mathrm{sec}$. The TRACE data show no time lag at the time scale of radiative or conductive cooling, but do show a much longer lag. Furthermore, the ratio of average $171 \AA$ to $195 \AA$ emission is inconsistent with any kind of superposition of cooling light-curves (Kankelborg and Longcope, 1999).

\section{Conclusion}

Comparing our theoretical model to observations suggests that magnetic reconnection is important for an XBP. Recall that the very existence of an XBP demonstrates the existence of topological reconnection (Harvey et al., 1994). EIT and MDI observations indicate that $P 01$ and N01 shared flux with closer sources at 19:00 on June 16, 1998, but were principally connected to one another by 7:00 on June 17. It is also significant that enhanced emission (i.e. the dotted contour at $10 \%$ of maximum) defines a region similar in size and shape to the separatrix outlining the entirety of domain P01N01 (the dashed curves). This clearly suggests that XBP emission originates from post-reconnection flux. Furthermore, the most brightly emitting plasma (i.e. the inner-most solid contour at $75 \%$ of maximum) is closest to the separator, where topological change has occurred most recently.

On the other hand, a model assuming energy dissipation only at the moment of, and at the site of, topological reconnection produces images different from observations in significant respects. One simple explanation of the difference is that plasma heating appears to persist in the postreconnection flux tube long after the instant its topology is changed. Many models of fast reconnection, particularly resistive models (Craig, 1994), describe a single mechanism performing both roles, flux transfer and energy dissipation. Following this reasoning our model assumed flux transfer and energy dissipation occurred at the same rate. If we instead imagine that the topological energy described by Eq. (1) is 
converted to heat gradually for some time after the topological reconnection event, then we can readily explain both the continued radiation of field lines, long since reconnected, and the overall surplus of emission in lines of relatively low temperature ( $\mathrm{Fe}$ IX and $\mathrm{Fe} \mathrm{XII}$ ).

In retrospect, there are several theoretical reasons to expect a gradual energy release following rapid flux transfer. Merely passing flux through the separator does not make it current-free; further reorganization and heating may continue within the reconnected flux, in order to more completely relax it. Also, the reconnection process will most likely disturb the plasma, exciting MHD oscillations and/or turbulence within the reconnected flux system. Gradual dissipation of all forms of non-thermal energy would naturally result in post-reconnection heating of the plasma.

Our conclusion echoes similar results concerning the heating of AR loops (Litwin and Rosner, 1993). In this case, observed loop life-times in excess of radiative cooling times suggest the presence of some persistent heating mechanism. In our study of an XBP it would seem that while fast magnetic reconnection can explain the flux transfer process, it is not responsible for dissipating energy in the plasma.

Acknowledgments. This is based upon work supported by the National Science Foundation under grant No. ATM-9733424, and by NASA under grant NAG5-7877.

\section{References}

Baum, P. J. and A. Bratenahl, Flux linkages of bipolar sunspot groups: a computer study, Solar Phys., 67, 245-258, 1980.

Biskamp, D., Magnetic reconnection via current sheets, Phys. Fluids, 29, 1520-1531, 1986

Biskamp, D., E. Schwarz, and J. F. Drake, Two-fluid theory of collisionless magnetic reconnection, Phys. Plasmas, 4, 1002-1009, 1997.

Cargill, P. J., Some implications of the nanoflare concept, ApJ, 422, 381393, 1994.

Cargill, P. J. and J. A. Klimchuk, A nanoflare explanation for the heating of coronal loops observed by Yohkoh, ApJ, 478, 799-806, 1997.

Chapman, S. and P. C. Kendall, Liquid stability and energy transformation near a magnetic neutral line: a soluble nonlinear hydromagnetic problem, Proc. Roy. Soc., A271, 435-448, 1963.

Craig, I. J. D., Current sheet formation and dissipation in general X-point topoligies, $A \& A, \mathbf{2 8 3}, 331-338,1994$.

Craig, I. J. D. and A. N. McClymont, Linear theory of fast reconnection at an X-type neutral point, ApJ, 405, 207-215, 1993.

Dungey, J. W., Cosmic Electrodynamics, Cambridge University Press, Cambridge, U.K., 1958.

Golub, L., A. S. Krieger, J. K. Silk, A. F. Timothy, and G. S. Vaiana, Solar X-ray bright points, ApJ, 189, L93-L97, 1974.

Golub, L., A. S. Krieger, J. W. Harvey, and G. S. Vaiana, Magnetic properties of X-ray bright points, Solar Phys., 53, 111-121, 1977.

Green, R. M., Modes of annihilation and reconnection in magnetic fields, in Stellar and Solar Magnetic Fields. Proc. IAU Symp. 22, edited by R. Lust, pp. 398-404, North-Holland, 1965.

Greene, J. M., Geometrical properties of three-dimensional reconnecting magnetic fields with nulls, JGR, 93, 8583-8590, 1988.
Harvey, K. L., K. To Strong, N. Nitta, and S. Tsuneta, Are X-ray bright points the signature of magnetic field reconnection?, in Solar Active Region Evolution: Comparing Models with Observations, edited by K. S. Balasubramaniam and G. W. Simon, pp. 377-388, A.S.P., 1994.

Hassam, A. B., Reconnection of stressed magnetic fields, ApJ, 399, 159$163,1992$.

Heyn, M. and V. Semenov, Rapid reconnection in compressible plasma, Phys. Plasmas, 3, 2725-2741, 1996.

Kankelborg, C. C. and D. W. Longcope, Forward modeling of the coronal response to reconnection in an X-ray bright point, Solar Phys., 190, 5977, 1999.

Kankelborg, C. C., A. B. C. Walker, II, R. B. Hoover, and T. W. J. Barbee, Observation and modeling of soft $\mathrm{x}$-ray bright points. I. Initial results, ApJ, 466, 529-536, 1996.

Kopp, R. A. and G. Poletto, Coronal heating by nanoflares: individual events and global energetics, ApJ, 418, 496-505, 1993.

Lau, Y.-T. and J. M. Finn, Three-dimensional kinematic reconnection in the presence of field nulls and closed field lines, ApJ, 350, 672-691, 1990.

Litwin, C. and R. Rosner, On the structure of solar and stellar coronae: loops and loop heat transport, ApJ, 412, 375-385, 1993.

Longcope, D. W., Topology and current ribbons: A model for current, reconnection and flaring in a complex, evolving corona, Solar Phys., 169, 91-121, 1996.

Longcope, D. W., A model for current sheets and reconnection in X-ray bright points, $A p J, \mathbf{5 0 7}, 433-442,1998$.

Longcope, D. W. and S. C. Cowley, Current sheet formation along three dimensional magnetic separators, Phys. Plasmas, 3, 2885-2897, 1996.

Parker, E. N., Sweet's mechanism for merging magnetic fields in conducting fluids, JGR, 62, 509-520, 1957.

Parker, E. N., Nanoflares and the solar x-ray corona, ApJ, 330, 474-479, 1988.

Petschek, H. E., Magnetic field annihilation, in AAS-NASA Symposium on the Physics of Solar Flares, edited by W. N. Hess, pp. 425-439, NASA, 1964.

Porter, L. J. and J. A. Klimchuk, Soft X-ray loops and coronal heating, ApJ, 454, 499-511, 1995.

Priest, E. R., C. E. Parnell, and S. F. Martin, A converging flux model on an X-ray bright point and an associated canceling magnetic feature, ApJ, 427, 459-474, 1994.

Scholer, M. and D. Roth, A simulation study on reconnection and smallscale plasmoid formation, JGR, 92, 3223-3233, 1987.

Shay, M. A., J. F. Drake, R. E. Denton, and D. Biskamp, Structure of the dissipation region during collisionless magnetic reconnection, $J G R, \mathbf{1 0 3}$, 9165, 1998

Sonnerup, B. U. O., Magnetic field re-connexion in a highly conducting incompressible fluid, J. Plasma Phys., 4, 161-174, 1970.

Sweet, P. A., The neutral point theory of solar flares, in Electromagnetic Phenomena in Cosmical Physics, edited by B. Lehnert, pp. 123-134, Cambridge University Press, Cambridge, U.K., 1958.

Syrovatskii, S. I., Formation of current sheets in a plasma with frozen-in strong magnetic field, Sof. Phys. JETP, 33, 933-940, 1971.

Ugai, M. and T. Tsuda, Magnetic field line reconnection by localized enhancement of resistivity. I. Evolution in a compressible MHD fluid, $J$. Plasma Phys., 17, 337-356, 1977.

Webb, D. F., S. F. Martin, J. W. Moses, and J. W. Harvey, The correspondence between X-ray bright points and evolving magnetic features in the quiet sun, Solar Phys., 144, 15-35, 1993.

D. W. Longcope (e-mail: dana@mithra.physics.montana.edu) and C. C. Kankelborg 